\title{
NIGERIA
}

\section{NIGERIAN INSTITUTE OF SOCIAL AND ECONOMIC RESEARCH}

\author{
PHB5, \\ University of Ibadan, \\ Ibadan, \\ Nigeria.
}

Founded: 1950

Director: Professor V.P. Diejomoah.

Objectives: To promote Nigerian development.

Funding: Government; consultancy for private sector.

Research interests: Applied research on Nigerian development; agricultural and industrial economics; public finance.

Regular publications: Annual Report.

Other publications: Monograph series. 